\title{
The facial massage reduced anxiety and negative mood status, and increased sympathetic nervous activity
}

\author{
Tomoko Hatayama, Shingo Kitamura, Chihiro Tamura, Mayumi Nagano and Koichiro Ohnuki \\ User Science Institute, Kyushu University, Fukuoka, Japan \\ (Received 20 October 2008; and accepted 24 October 2008)
}

\begin{abstract}
The aim of this study was to clarify the effects of $45 \mathrm{~min}$ of facial massage on the activity of autonomic nervous system, anxiety and mood in 32 healthy women. Autonomic nervous activity was assessed by heart rate variability (HRV) with spectral analysis. In the spectral analysis of HRV, we evaluated the high-frequency components (HF) and the low- to high-frequency ratio ( $\mathrm{LF} / \mathrm{HF}$ ratio), reflecting parasympathetic nervous activity and sympathetic nervous activity, respectively. The State Trait Anxiety Inventory (STAI) and the Profile of Mood Status (POMS) were administered to evaluate psychological status. The score of STAI and negative scale of POMS were significantly reduced following the massage, and only the LF/HF ratio was significantly enhanced after the massage. It was concluded that the facial massage might refresh the subjects by reducing their psychological distress and activating the sympathetic nervous system.
\end{abstract}

Body massage treating large muscles located in upper limb, shoulders and back brings many psychological and physiological effects such as decreasing anxiety and depression, increasing attentiveness, relieving stress and pain, and reducing blood pressure $(1-3,10,13)$.

In the case of facial massage, a popular cosmetic technique preferred by women, much anecdotal evidence has been reported that facial massage can enhance relaxation just as well as body massage. However, very few studies have provided scientific evidence, such as physiological or psychological data. To date, only two studies $(7,17)$ have reported the change in psychological parameters and electroencephalogram (EEG) data following aesthetic facial massage. They observed higher subjective scores in both general deactivation and deactivation-sleep factors, significant reduction of anxiety, and a marked attenuation of the alpha-waves accompanied by a small increase in theta-waves during massage, an ef-

Address correspondence to: Tomoko Hatayama User Science Institute, Kyushu University 4-9-1 Shiobaru, Minami-ku, Fukuoka Japan 815-8540 Tel \& Fax: +81-92-553-9458

E-mail: hata@usi.kyushu-u.ac.jp fect which they attributed to sleep induction by facial massage. Therefore, facial massage might have positive effects not only on beauty, but also on psychological status or cerebral activity.

In the case of EEG, often measured in preceding studies, it is difficult to avoid equipment noises and the misalignment of electrodes in treating facial muscles. Analysis of autonomic nervous activity using heart rate variability derived from electrocardiogram (ECG) through a chest lead is available as an alternative physiological measurement. However, there have been no studies evaluating the possible effect of facial massage using such a method. Furthermore, even though Inoue et al. (6) and Hughes and Stoney (5) reported that changes in mood state were associated with autonomic nervous system activity, no study has investigated the effects of facial massage from both physiological and psychological aspects.

The present study therefore tested the following 2

This research was undertaken as outsourced work from the Ministry of Education, Culture, Sports, Science and Technology (science and technology advancement adjustment expense; referred to as Kyushu University User Science Institute). 
hypotheses;

1) There will be an enhancement of physiological relaxed status in the activity of autonomic nervous system assessed using a power spectral analysis of heart rate variability following a facial massage.

2) There will be a reduction in psychological distress as evaluated using the Profile of Mood States (POMS) and the State Trait Anxiety Inventory (STAI) following a facial massage.

\section{MATERIALS AND METHODS}

Subjects. Data was collected from 32 healthy Japanese women ranging in age from 20 to 40 years (mean, 28.5 years). After each subject had been briefed on the objective and method of the experiment, written informed consent was obtained.

Protocol. Measurements were carried out before and after a facial massage in a private room set at an ambient temperature average $26.8 \pm 1.2^{\circ} \mathrm{C}$. The facial massage consisted of four steps: (1) cleansing with cleansing cream by hand, (2) removal of keratin with steaming, (3) ultrasonic wave facial treatment with gel, and (4) application of lotion for moisture retention. The total time for this treatment was $45 \mathrm{~min}$ and it was carried out by trained, experienced cosmetologists.

The subjects were first asked to complete the POMS-short form and STAI-JYZ in sitting position, keeping the electrodes on their chests. The ECG was recorded for $10 \mathrm{~min}$ both before and after the massage, with subjects lying on their backs. The data obtained between third to sixth minute was used for power spectral analysis. The details of measurement are described below.

Electrocardiogram procedures. ECG was recorded with MWM-01 monitor (GMS Inc, Tokyo, Japan) and was analyzed by the MemCalc system (MemCalc/Makin2; GMS Inc) on an on-line computer (VAIO, VGN-T70B; Sony Co., Tokyo, Japan).

ECG was recorded from electrodes placed in the CM5 position. In order to evaluate cardiac autonomic nervous activity, a power spectral analysis of the temporal intervals between heart beats (R-R intervals) was used. Power spectral analyses using the MemCalc system were performed for each 5 second period. We obtained the R-R intervals (ms) as the time domain index of heart rate variability (HRV). The power of HRV was quantified by determining the areas of the spectrum in 2 component widths: low frequency (LF, 0.04-0.15 Hz) and high frequency $(\mathrm{HF}, 0.15-0.4 \mathrm{~Hz})$. The ratio of LF power to HF power (LF/HF ratio) was also assessed. The HF component represents parasympathetic nervous activity $(11,12)$ while the LF/HF component is a representation of sympathetic nervous activity $(8,18)$.

POMS-short form. POMS-short form, which had been shortened and translated into Japanese (19), was administered. The POMS-short form comprised of 30 questions about the current mood state. These 30 questions were classified in 6 sub-scales: T-A (tension and anxiety), D (depression and dejection), $\mathrm{A}-\mathrm{H}$ (anger and hostility), V (vigor), F (fatigue), and $\mathrm{C}$ (confusion). The subjects selected the raw score from one of five values $(0,1,2,3$ and 4 , where $0=$ no such mood state and $4=$ extreme mood state). These raw scores in each sub-scale were then added to generate each sub-scale score.

STAI-JYZ. The STAI (15) is a 20-item scale that measures acute level of anxiety. The subjects selected the raw score from one of four values $(1,2,3$ and 4 , where $1=$ not at all and $4=$ very much). A summary score is obtained by adding the weight of each item. The STAI scores indicate an increase in response to situational stress and a decline under relaxing conditions. In this study, STAI-JYZ, which regards Japanese cultural factors better, was used. The STAI-JYZ exhibits acceptable internal consistency and test-retest reliability (4).

Statistical analysis. Each data was expressed as means \pm standard error (SE). Student's paired $t$ test was used to evaluate, and statistics were calculated with SPSS version 14 (SPSS Inc. Chicago, Illinois, USA). Results were considered significance at $P<$ 0.05 .

\section{RESULTS}

LF/HF ratio remarkably increased from $0.81 \pm 0.11$ to $1.22 \pm 0.19$ after the massage (Fig. $1, P=0.035$ ), even though the HF power and heart rate remained unchanged (data not shown).

Fig. 2 shows the differences in average scores between pre-massage and post-massage for the six subscales of the POMS test. Most scores, which indicate negative feelings, tended to decrease after the massage. Especially, T-A (tension and anxiety) significantly declined from $3.66 \pm 0.62$ to $1.16 \pm 0.33$, and also $\mathrm{F}$ (fatigue) dropped $2.19 \pm 0.58$ to $0.81 \pm$ $0.24(P<0.001)$. The anger and vigorous scores 
showed no significant changes.

Similarly, the score for anxiety in the STAI significantly declined from $36.25 \pm 1.33$ to $28.25 \pm 1.04$ following the massage $(P<0.001)$.

\section{DISCUSSION}

To date, many studies have reported positive psychological and physiological effects of body massage when performed as a method of psychosomatic conditioning. However, very little research exists on aesthetic facial massage, which is usually performed expecting not psychosomatic treatment but rather beautification of facial skin. We therefore examined the effects of facial massage from both physiologi-

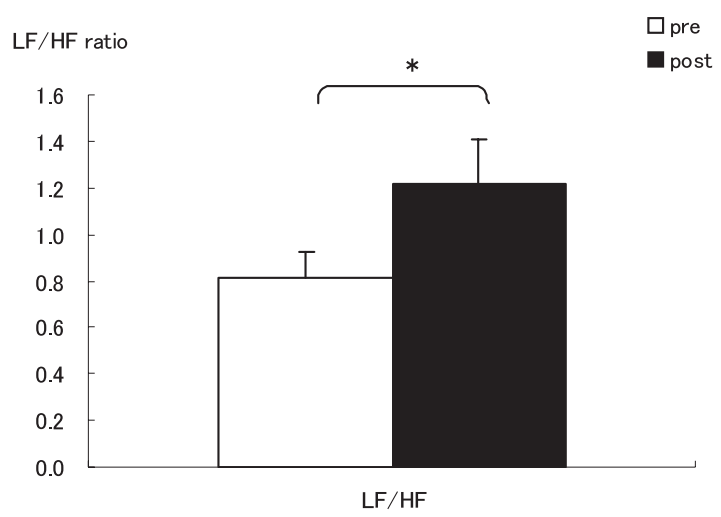

Fig. 1 Change of LF/HF ratio before and after facial massage. Error bars indicate $\pm \mathrm{SE}, \mathrm{n}=32,{ }^{*}: P<0.05$ cal and psychological aspects, by evaluating autonomic nervous activity and mental status.

Anxiety in the STAI and negative moods in POMS were significantly decreased following the massage. These results are consistent with previous findings $(10,13)$. In the present study, the score of the STAI and the negative scales of POMS at baseline were slightly lower as compared with the corresponding normal values for healthy women cited in the guidelines of Japanese version of the STAI and POMS. Despite relatively low negative psychological scores before the facial massage, the scores following facial massage significantly decreased, as well as body massage. These results suggested that the facial massage had strong effects on stress alleviation or psychological relaxation.

Contrary to our prediction, sympathetic nervous activity increased following the massage. It is speculated that the discrepancy was simply due to differences in the timing of measurement. According to preceding studies, heart rate and LF/HF ratio declined during the massage, but these affects almost subsided shortly after massage $(13,16)$. It is not surprising that parasympathetic nervous activity was enhanced during the massage, because the subjects stayed in a relaxed position or their eyes closed during the massage.

Selye (14) once announced that a stressor, which was a stimulus inducing a stress reaction to biological body, could not only be distress but also eustress. Eustress contains positive stimuli such as

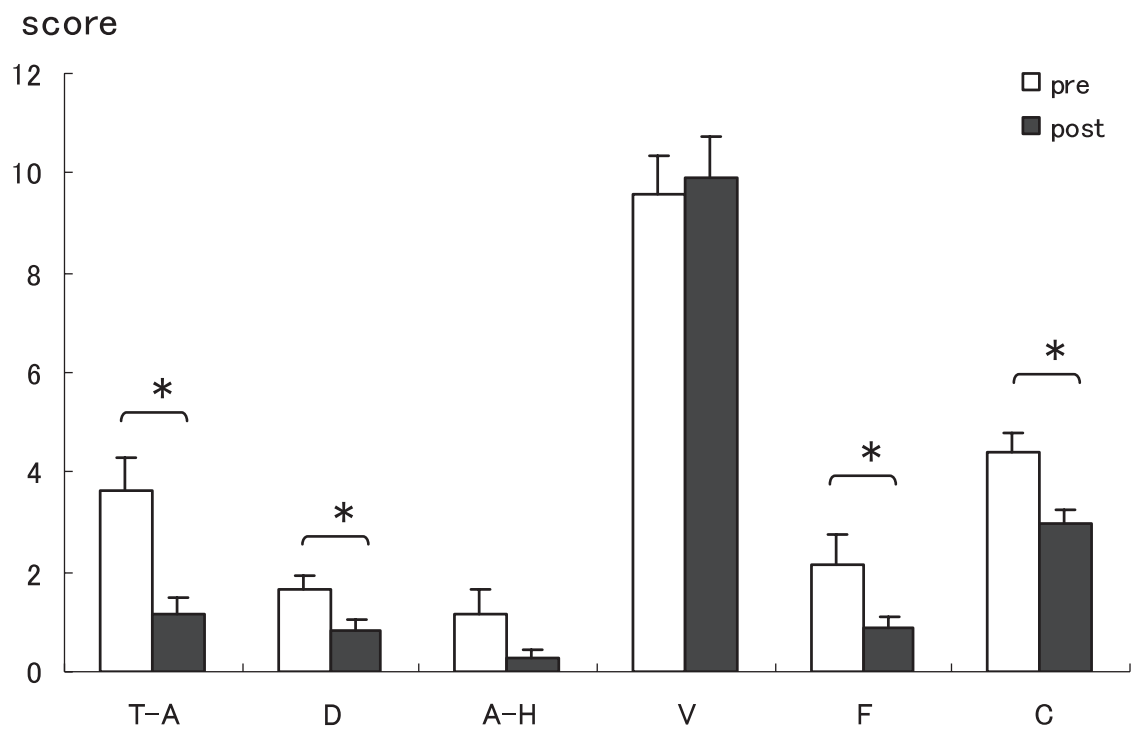

Fig. 2 Changes in mean POMS test score before and after facial massage. Each value represents the mean score for six sub-scales of the POMS test, and is expressed as the mean $\pm \mathrm{SE}\left(\mathrm{n}=32,{ }^{*}: P<0.05\right)$. T-A (tension and anxiety), D (depression and dejection), A-H (anger and hostility), V (vigor), $\mathrm{F}$ (fatigue), and $\mathrm{C}$ (confusion). 
taking a bath, deep or sweet sleep, mental satisfaction, and so on (9). It was thus speculated that the increased sympathetic nervous activity following the facial massage in the present study might be a positive stress reaction to a stressor which is classified as eustress. Taking these results into consideration, such a mixed status of psychologically relaxed and physically-activated might well be regarded as refreshment rather than relaxation.

Heart rate after facial massage did not change compared to pre-massage values in the present study. It was guessed that the facial massage treating relatively-small facial muscles did not change the circulation as much as heart rate was affected.

In conclusion, the present study could demonstrate that facial massage reduces psychological distress and activates the subjects physically. Taken together, it might be reasonable that facial massage originally performed as a cosmetic treatment has an effect of refreshment rather than relaxation. Thus, it is expected that using psychological and physiological evaluation methods simultaneously enables us to extract more appropriate information and better assess the effects of various stimuli.

The present study had several limitations, such as the lack of measurement during the massage period and a control group. Further study should be designed as a randomized controlled trial, and the data should be continuously collected through the experiment.

\section{Acknowledgements}

This research was financially supported by Tina-pri Corporation. We thank the massage therapists and the assistants who helped with this research.

\section{REFERENCES}

1. Diego MA, Field T, Sanders C and Hernandez-Reif M (2004) Massage therapy of moderate and light pressure and vibrator effects on EEG and heart rate. Int $J$ Neurosci 114, 31-44.

2. Field T, Morrow C, Valdeon C, Larson S, Kuhn C and Schanberg S (1992) Massage reduces anxiety in child and adolescent psychiatric patients. J Am Acad Child Adolesc Psychiatry 31, 125-131.

3. Field T, Ironson G, Scafidi F, Nawrocki T, Goncalves A, Burman I, Pickens J, Fox N, Schanberg S and Kuhn C (1996)
Massage therapy reduces anxiety and enhances EEG pattern of alertness and math computations. Int J Neurosci 86, 197205.

4. Hidano T, Fukuhara M, Iwawaki M, Soga S and Spielberger CD (2000) New STAI Manual State-Trait Anxiety InventoryForm JYZ. Jitsumu Kyouiku Press, Tokyo. (in Japanese)

5. Hughes JW and Stoney CM (2000) Depressed mood is related to high-frequency heart rate variability during stressors. Psychosom Med 62, 796-803.

6. Inoue N, Kuroda K, Sugimoto A, Kakuda T and Fushiki T (2003) Autonomic nervous responses according to preference for the odor of Jasmine tea. Biosci Biotechnol Biochem 67, $1206-1214$.

7. Jodo E, Yamada Y, Hatayama T, Abe T and Maruyama K (1988) Effects of facial massage on the spontaneous EEG. Tohoku Psychologica Folia 47, 8-15.

8. Koizumi K, Terui N and Kollai M (1985) Effect of cardiac vagal and sympathetic nerve activity on heart rate in rhythmic regulation. J Auton Nerv Syst 12, 251-259.

9. Lazarus RS and Folkman S (1984) Stress, Appraisal, and Coping. Springer Publishing Company, Inc, New York.

10. McNamara ME, Burnham DC, Smith C and Carroll DL (2003) The effects of back massage before diagnostic cardiac catheterization. Altern Ther Health Med 9, 50-57.

11. Pagani M, Lombardi F, Guzzetti S, Rimoldi O, Furlan R, Pizzinelli P, Sandrone G, Malfatto G, Dell'Orto S, Piccaluga E, Turiel M, Baselli G, Cerutti S and Malliani A (1986) Powerspectral analysis of heart rate and arterial pressure variabilities as marker of sympathovagal interaction in man and conscious dog. Circ Res 59, 178-193.

12. Akselrod S, Gordon D, Ubel FA, Shannon DC, Berger AC and Cohen RJ (1981) Power spectrum analysis of heart rate fluctuation: a quantitative probe of beat-to-beat cardiovascular control. Science 213, 220-222.

13. Sato T (2006) Effects of hand massage on the human autonomic system and mood in healthy women. Yamanashi Nursing Journal 4, 25-32. (in Japanese with English abstract)

14. Selye H (1974) Stress Without Stress. Lippincott, Philadelphia.

15. Spielberger CD, Gorusch TC and Lushene RE (1970) The State Trait Anxiety Inventory. Consulting Psychologists Press, Palo Alto, CA.

16. Tochikubo O, Ri S and Kura N (2006) Effects of pulsesynchronized massage with air cuffs on peripheral blood flow and autonomic nervous system. Circ J 70, 1159-1163.

17. Yamada Y, Hatayama T, Maruyama K, Abe T and Suzuki Y (1986) A psychological effect of facial estherapy. Tohoku Psychologica Folia 45, 6-16.

18. Yeragani VK, Srinivasan K, Vempati S, Pohl R and Balon R (1993) Fractal dimension of heart rate time series: an effective measure of autonomic function. $J$ Appl Physiol 75, 2429-2438.

19. Yokoyama K (2005) POMS-short form handbook and case study. Kaneko Shobo, Tokyo. (in Japanese) 